\title{
Structure of White Light Emitting CdSe Nanocrystals
}

\author{
T.J. Pennycook, ${ }^{*, *}$ J.R. McBride, ${ }^{* * *}$ S.J. Rosenthal, ${ }^{* * * *}$ S.T. Pantelides, ${ }^{* * *}$ and S.J. Pennycook ${ }^{* * *}$ \\ * Department of Physics and Astronomy, Vanderbilt University, Nashville, TN 37235 \\ ** Materials Science and Technology Division, Oak Ridge National Laboratory, PO Box 2008, Oak \\ Ridge, TN 37831-6071 \\ *** Department of Chemistry, Vanderbilt University, Nashville, TN 37235
}

Replacing conventional lighting with far more efficient solid-state lighting could reduce global electricity needs enormously [1], but achieving an appealing white light has been challenging. In 2005, however, a method to produce pure white light from a light emitting diode (LED) was developed. An ultraviolet LED is coated in ultrasmall CdSe nanoclusters (NCs) around $2 \mathrm{~nm}$ in diameter which absorb the ultraviolet and reemit pure white light [2]. Since then much work has been devoted to increasing the quantum efficiency of this process by adjusting the growth process. Efficiencies as high as $9 \%$ have now been achieved. The physical process behind the white light emission has however remained elusive. We use a combination of scanning transmission electron microscopy (STEM) and density functional theory (DFT) to search for the underlying source of white light emission in CdSe NCs.

Quantum confinement allows one to tune the band gaps of semiconductor NCs. As a result, by changing the size of a $\mathrm{NC}$ it is possible to also change the frequency at which it emits light For example, using $\mathrm{CdSe} / \mathrm{ZnS}$ core/shell NCs with different sizes one can obtain monochromatic emission across the visible range, as shown in Fig. 1. The smallest NCs produce the highest energy emission, and a high resolution image of a blue-light-emitting $\mathrm{CdSe} / \mathrm{ZnS}$ core/shell $\mathrm{NC}$ is shown in Fig. 1(a) revealing its crystalline nature. The CdSe core of this $\mathrm{NC}$ was grown to be $2 \mathrm{~nm}$ or less, which appears likely from the image. Although pure CdSe NCs can be produced which emit monochromatic red to green light, the synthesis of smaller CdSe NCs results not in blue, but white light emission. The transition to white light emission occurs at a nominal size of around $2 \mathrm{~nm}$.

High resolution aberration corrected STEM reveals that this size corresponds to a structural transition: CdSe NCs larger than $2 \mathrm{~nm}$ are clearly seen to be stable wurtzite crystallites such as the $3 \mathrm{~nm}$ cluster shown in Fig. 1(b), but those $2 \mathrm{~nm}$ and smaller appear to be in state of continuous structural flux. We have recorded high-resolution ADF movies of these ultrasmall NCs, and a representative series of frames from one such movie is shown in Fig. 2. The structure continually morphs from one shape to another, and no consistent crystal structure is apparent. Using accelerating voltages of 60,100 and $300 \mathrm{kV}$ as well as beam currents varying up to a factor of five results in no perceivable changes in the rate at which the structures morph. Furthermore the clusters appear deformed and often surrounded by many free atoms the moment they are imaged. DFT simulations show that thermal motion causes small CdSe NCs to morph in ways remarkably similar to those seen on the microscope. Snapshots from such a simulation are shown in Fig. 3. We will present further STEM and DFT investigations into the effects of this fluxionallity on the electronic structure of ultrasmall CdSe NCs. 
References

[1] S. Nakamura, MRS Bulletin 34, 101 (2009).

[2] M.J. Bowers II et al., J. Am. Chem. Soc. 127, 15378 (2005).

[3] J.R. McBride et al., Chem. Phys. Lett., 498, 1 (2010).

[4] J.R. McBride et al., Nano Lett. 4, 1279 (2004).

[5] Research supported by the DOE Office of Basic Energy Sciences, Materials Science and Engineering Division, DOE grant DE-FG02-09ER46554, the McMinn Endowment, NERSC.
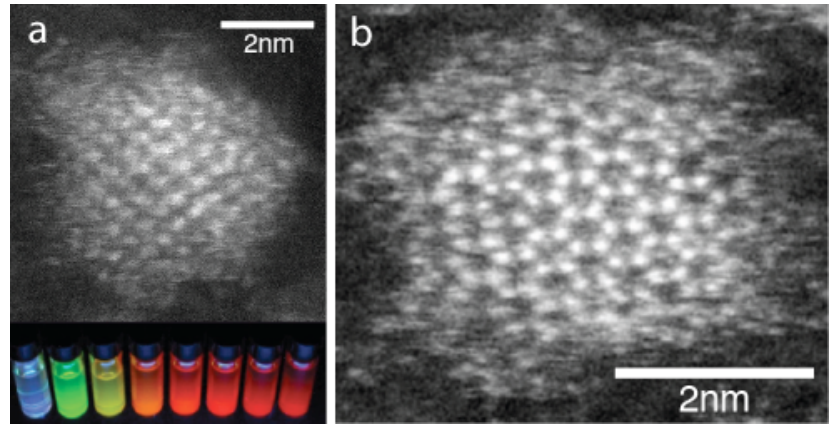

FIG. 1. High resolution ADF images of (a) a blue-light-emitting $\mathrm{CdSe} / \mathrm{ZnS}$ core/shell $\mathrm{NC}$ and (b) a $3 \mathrm{~nm}$ CdSe NC (adapted from [3]). The inset to (a) shows suspensions of CdSe core NCs with sizes from 2 to $6 \mathrm{~nm}$ emitting in colors spanning the visible spectrum.
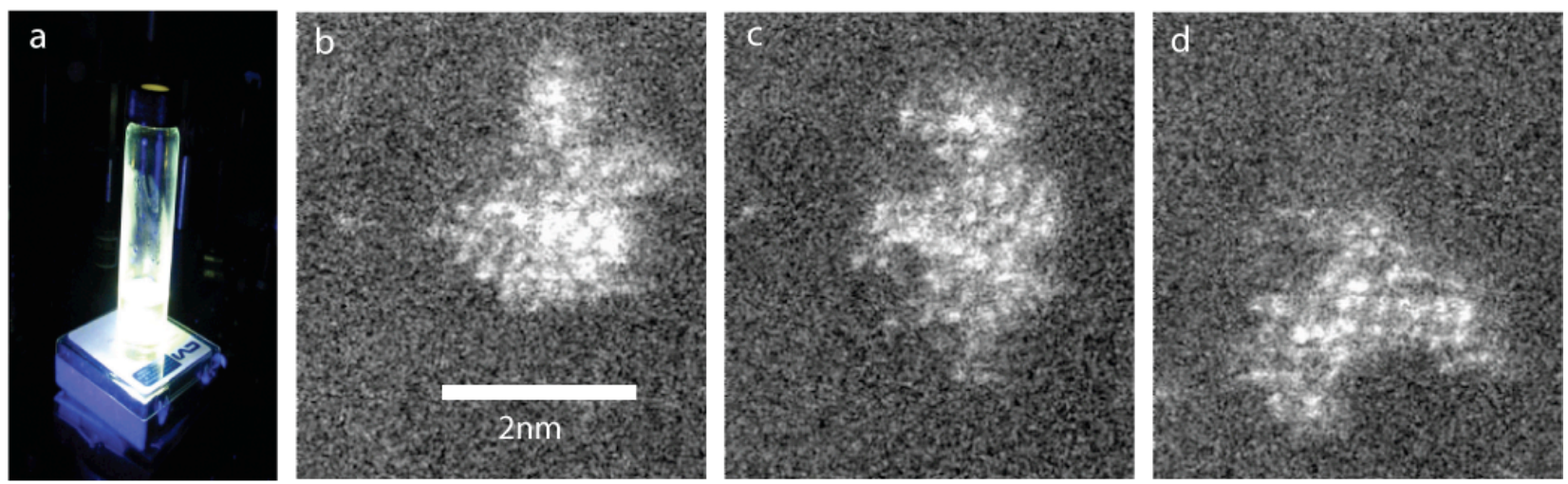

FIG. 2. (a) White light emission from ultrasmall CdSe NCs. (b-d) Frames from a high resolution ADF movie of an ultrasmall CdSe NC.

a

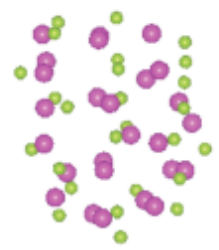

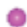

b

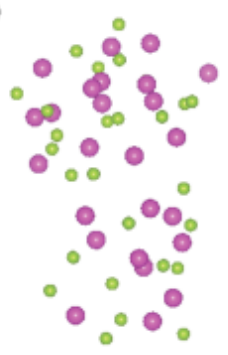

C

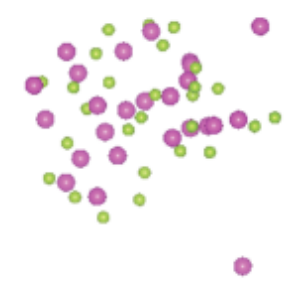

FIG. 3. Snapshots of a $\mathrm{Cd}_{27} \mathrm{Se}_{27} \mathrm{NC}$ during a finite temperature DFT MD simulation showing how ultrasmall NCs can morph. 